\title{
Magnetic Measurements of the Wedge-Pole Prototype Undulator
}

\section{Vasserman, J. Pflüger, and E. Gluskin Experimental Facilities Division}

\section{DISCLAIMER}

This report was prepared as an account of work sponsored by an agency of the United States Government. Neither the United States Government nor any agency thereof, nor any of their employees, makes any warranty, express or implied, or assumes any legal liability or responsibility for the accuracy, completeness, or usefulness of any information, apparatus, product, or process disclosed, or represents that its use would not infringe privately owned rights. Reference herein to any specific commercial product, process, or service by trade name, trademark, manufacturer, or otherwise does not necessarily constitute or imply its endorsement, recommendation, or favoring by the United States Government or any agency thereof. The views and opinions of authors expressed herein do not necessarily state or reflect those of the United States Government or any agency thereof.

\section{$6 / 25 / 93$}

\section{Advanced Photon Source Argonne National Laboratory}

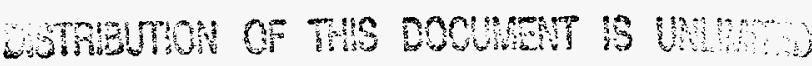
$111^{3}$

RECENED

JUL 251935 


\section{DISCLAIMER}

Portions of this document may be illegible in electronic image products. Images are produced from the best available original document. 
MAGNETIC MEASUREMENTS OF THE WEDGE-POLE PROTOTYPE UNDULATOR

\section{TABLE OF CONTENTS}

1. Introduction

2. Magnetic Measurement Technique

2.1 Hall Probe

2.2 Search Coils

3. Performance Evaluation of the Wedge-Pole Undulator

3.1 Hall Probe Measurements

3. Coil Measurements

4. Conclusion 


\section{INTRODUCTION}

The Advanced Photon Source (APS), a 7-GeV positron storage ring, will have as many as 34 straight sections available for installation of multiple insertion devices (IDs). The size and the taper of the gap of the IDs will be able to be varied to tune the energy of the emitted radiation to the experiments. The IDs must not affect the particle beam motion. Therefore, the disturbances should be kept within tight boundaries to assure that the ring performance parameters are maintained and that the emitted radiation shows little degradation from an ideal device. These requirements are derived from the stability criteria of the beam to be stable within $10 \%$ of the emittance [1]. The requirements cannot be met without using closed loop orbit compensation. With such a system installed, it is reasonable to relax the requirements on the ID magnetic field performance. These relaxed requirements are listed in Table 1 for integrated multipole moments.

\section{Table 1. Integrated Multipole Moments}

Multipole $n$ 1(Quadrupole) 2(Sextupole) 3 (Octupole)

Normal component $\left(\mathrm{b}_{n}\right)$
50 Gauss
200 Gauss $/ \mathrm{cm}^{2}$
300 Gauss $/ \mathrm{cm}^{2}$

Skew component $\left(a_{n}\right)$
50 Gauss
100 Gauss $/ \mathrm{cm}^{2}$
50 Gauss $/ \mathrm{cm}^{2}$

We use the common definition of the multipole moments:

$$
\int\left(B_{y}+i B_{x}\right) d z=\Sigma\left(b_{n}+i a_{n}\right)(x+i y)^{n} \text {, }
$$

where $x$ and $y$ are transverse horizontal and vertical space coordinates; $B_{x}$ and $B_{y}$ are the horizontal and vertical magnetic fields, respectively; $b_{n}$ and $a_{n}$ are the normal and skew components of the integrated multipole moments of the field; and $z$ corresponds to the longitudinal direction of the undulator.

It is impossible to use scanning along the y-direction due to a small gap size. Therefore, we chose to scan along the $x$-direction in the median plane $(y=0)$. From the above expression, we obtain equations for determination of normal and skew components of the integrated multipole moments of the field in this case : 


$$
\begin{aligned}
& \int_{-\infty}^{\infty} d_{z} B_{y}=\sum_{0}^{N} b_{n} x^{n} \\
& \int_{-\infty}^{\infty} d_{z} B_{x}=\sum_{0}^{N} a_{n} x^{n}
\end{aligned}
$$

Polynomial fitting to the measured data of the integrated fields up to the 3rd order $(\mathrm{N}=3)$ as a function of $x$ results in multipole coefficients $a_{n}$ and $b_{n}$, as well as their errors (see, e.g., [2]). Scanning was done in the required "good field" region $( \pm 5 \mathrm{~mm}$ in the $x$-direction).

The first vertical and horizontal field integrals are required to be $\leq 100$ Gauss-cm. The second vertical and horizontal field integrals are required to be $\leq 100,000$ Gauss- $\mathrm{cm}^{2}$. Currently, magnetic measurement tests have been performed on a wedge-pole undulator to examine the quality of the undulator prototype and to test the magnetic measurement facility.

Two types of undulators were examined. The symmetric undulator has an integer number of periods and both end poles of equal strength. The antisymmetric undulator has a half-integer number of periods, and the downstream and upstream end strengths are of opposite sign. The magnetic measurement facility is described in detail in [1].

The first and second field integrals are defined:

$$
\begin{aligned}
& J_{1}(l)=\int_{-}^{l} B(z) d z \\
& J_{2}(l)=\int_{-}^{l} J_{1}(z) d z,
\end{aligned}
$$

where $B(z)$ is the horizontal or vertical magnetic field for the horizontal or vertical field integrals, respectively.

\section{Magnetic Measurement Technique}

There are two types of magnetic sensors available for the magnetic measurement system, the Hall sensor and the moving search coils.

\subsection{Hall Probe Sensor}

A Hall probe (model MPT-141) from Group3 is currently used. The . Hall probe is miniature sized with a temperature sensor built into the probe head. The probe has the overall dimensions of $5 \times 14 \times 2$ $\mathrm{mm}$ with a sensitive area of $1 \times 0.5 \mathrm{~mm}$ encapsulated in epoxy and mounted on a reference surface made of ceramic. The probe is connected to a Hall probe interface (HPI) with a low thermal drift. 
The Hall probe also has a low thermal drift and was accurately calibrated at given temperatures to provide a calibration chart of its voltage response to the strength of the magnetic field. The measured voltage is converted to a magnetic field using a fitting function based on the calibration table.

\subsection{Search Coils}

Four coils have been used in the characterization of the wedge-pole ID (see Table 2 for coil parameters).

Table 2

\begin{tabular}{|l|l|l|l|l|}
\hline Coil \# & $\begin{array}{l}\text { Measured } \\
\text { field }\end{array}$ & $\begin{array}{l}\text { length } \\
\mathrm{m} \mathrm{m}\end{array}$ & $\begin{array}{l}\text { width } \\
\mathbf{m} \mathbf{~ m}\end{array}$ & \# of turns \\
\hline 1 & Vertical & 33. & 10. & 100 \\
\hline 2 & Vertical & 510. & 7. & 170 \\
\hline 3 & Horizontal & 16.5 & 4. & 400 \\
\hline 4 & Horizontal & 165. & 4. & 400 \\
\hline
\end{tabular}

\section{Performance Evaluation of the Wedge-Pole Undulator}

The wedge-pole undulator is a planar insertion device with a period length of $3.3 \mathrm{~cm}$ and 25 full-strength magnetic periods (the same period length as Undulator $A$ ). The magnetic field reaches a maximum of 0.7 Tesla at the minimum gap of $11.5 \mathrm{~mm}$. The transition to the antisymmetric end pole configuration was implemented by making a half-integer number of magnetic periods $(24.5$, e.g.), that corresponds to an even number of poles.

\subsection{Hall Probe Measurements}

The miniature Group3 Hall probe and Hall probe interface (HPI) were used for magnetic tests of the wedge-pole undulator. The first results were obtained for the regular symmetric-end-pole undulator.

The wedge-pole magnetic field and first and second vertical field integrals were measured. The accuracy of the Hall probe system was investigated with respect to the required specifications based on analysis of these scans.

The main factor limiting the accuracy of the Hall probe measurements is the noise of the HPI system. The dependence of the Hall probe noise level on the integration time is shown in Fig. 1 (for integration times of $100 \mathrm{~ms}, 33 \mathrm{~ms}$, and $7 \mathrm{~ms}$ ). For most of the 
Hall probe measurements, an integration time of $33 \mathrm{~ms}$ was chosen as a compromise between measuring time and noise level.

A second important factor for the accuracy of Hall probe measurement is the temperature instability. The vendor data show the variation of .5 Gauss $/{ }^{\circ} \mathrm{C}$. Our measurements in a free space out of magnetic field show the temperature dependence of the first field integral equal to $15 \mathrm{G}-\mathrm{cm} /{ }^{\circ} \mathrm{C}$ at a distance $80 \mathrm{~cm}$, thus less than $\pm .1{ }^{\circ} \mathrm{C}$ temperature stability is required so that the value of the systematic errors in the measurements are comparable to the statistical RMS errors.

A set of Hall probe measurements was performed to estimate the value of the integrated multipole moments of the wedge-pole undulator (normal components). For this purpose, we investigated the first vertical field integral dependence on the $x$-coordinate in the median plane. The result is shown in Fig. 2 for a gap of $11.5 \mathrm{~mm}$.

The results of a third order polynomial fit are shown in Table 3 for the regular symmetric-end-pole undulator. Only the dipole component does not satisfy the requirement of being less than 100 G-cm. Many different techniques were attempted in this set of the measurements to bring the first and second field integrals to the desired values. One possible solution is shimming the end poles in order to zero the first field integral for the chosen gap, but the difference in the first field integrals for all ranges of gaps is rather large, approximately equal to 200 Gauss-cm. A transition to antisymmetric-end-pole geometry sufficiently decreases this value.

Table 3. Integrated Multipole Moments

\begin{tabular}{|l|l|}
\hline Multipole $\mathrm{n}$ & Normal Component $(\mathrm{bn}$ \\
\hline 0 (dipole) & $360 \mathrm{G}-\mathrm{cm} \pm .9$ \\
\hline 1 (quadrupole) & $-1.5 \mathrm{G} \pm 4.8$ \\
\hline 2 (sextupole) & $-5 \mathrm{G} / \mathrm{cm}^{\prime} \pm 6.6$ \\
\hline 3 (octupole) & $-43 \mathrm{G} / \mathrm{cm}^{2} \pm 24.8$ \\
\hline
\end{tabular}

The results of measurements of the gap dependence for the first vertical field integral in both cases are shown in Fig. 3. Curves A and $B$ correspond to different shimming in the symmetric case. The results of measurements of the first and second vertical field integral dependence on the gap for $(x, y)=(0,0)$ in the antisymmetric case are shown in Table 4. 
Table 4. Antisymmetric End Pole Results (23.5 periods)

\begin{tabular}{|c|c|c|}
\hline $\operatorname{Gap}(\mathrm{mm})$ & $\mathrm{I}_{1}$ (Gauss-cm) & $\mathrm{I}_{2}$ (Gauss-cm ${ }^{2}$ ) \\
\hline 50. & 28 & -20000 \\
\hline 35. & 26 & -18800 \\
\hline 28. & 46 & -14360 \\
\hline 20. & 37 & -5070 \\
\hline 15.5 & 46 & 6820 \\
\hline 13.5 & 59 & 15320 \\
\hline$\overline{11.5}$ & $\overline{87}$ & 25000 \\
\hline
\end{tabular}

Results for the first field integral in the antisymmetric case are rather satisfactory. Second field integral values for the undulator with a length of $2.4 \mathrm{~m}$ could be extrapolated roughly by multiplying these values by a factor of three, and they are very close to the requirements. The results of typical field map measurements in the antisymmetric and symmetric configurations are shown in Figs. 4-6.

The Hall probe data were obtained with RMS errors equal to 2-5 Gauss-cm. The latest improvements in measurement technique decreased the average RMS errors up to $2 \mathrm{G}-\mathrm{cm}$. This accuracy was obtained when no zero-field measurements were performed before each scan. The Hall probe zero value was determined once before each set of measurements by averaging over the time equal to the usual measurement time. (Hall probe zero drift time is much larger than measurement time.)

The next set of Hall probe measurements was of the integrated multipole moments for different wedge-pole gap settings in the antisymmetric configuration. The results of a third order polynomial fit are shown in Table 5

Table 5. Integrated Multipole Moments (Normal Components)

\begin{tabular}{|l|c|c|c|c|}
\hline gap $(\mathrm{mm})$ & $\mathrm{b}_{0}(\mathrm{G}-\mathrm{cm})$ & $\mathrm{b}_{1}(\mathrm{G})$ & $\mathrm{b}_{2}(\mathrm{G} / \mathrm{cm})$ & $\mathrm{b}_{3}\left(\mathrm{G} / \mathrm{cm}^{2}\right)$ \\
\hline 11.5 & $119 . \pm .55$ & $25.5 \pm 3$. & $-20.4 \pm 4.1$ & $-32.7 \pm 15.4$ \\
\hline 14.5 & $106 . \pm 1.4$ & $8.1 \pm 7.4$ & $-21.7 \pm 10.3$ & $-28.7 \pm 38.3$ \\
\hline 20. & $72.4 \pm 1.2$ & $-7 . \pm 6.4$ & $-18.5 \pm 8.9$ & $23.6 \pm 33$. \\
\hline 27. & $54 . \pm .9$ & $-4.5 \pm 5.1$ & $-2.95 \pm 7.1$ & $-3 . \pm 26.4$ \\
\hline 35. & $38.5 \pm .9$ & $-18.9 \pm 5$. & $3.66 \pm 7.1$ & $9.49 \pm 26.5$ \\
\hline 51. & $36.8 \pm 1.5$ & $-33 . \pm 10$. & $7.2 \pm 14$. & $13.2 \pm 69.1$ \\
\hline
\end{tabular}

The RMS error values of the measurements cannot be explained solely by statistical errors and the HPI-produced noise. If they could, the accuracy would improve by increasing the measurement 
time. But it does not, indicating the existence of some systematic errors. The cause of such errors is not clear and should be investigated. It certainly is not a result of temperature fluctuations, because this dependence was too small to produce such values. But the current accuracy is good enough to obtain all the necessary results with a Hall probe.

Field map measurements were performed away from the wedgepole undulator to separate the effects of Hall probe measurement errors and other sources. The results are shown in Fig. 7. The typical time between consecutive measurements is approximately one day.

\subsection{Coil Measurements}

Different coils were used for the first set of wedge-pole measurements, see Section 2.2 for coil descriptions. $B_{y}$ coils were used for the first vertical field integral measurements to check the results of Hall probe measurements. $B_{\mathbf{X}}$ coils were used for the first horizontal field integral measurements. The $B_{\mathbf{X}}$ coil was also used to study the dependence of the first horizontal field integral on the horizontal coordinate. In contrast to the Hall probe system, the integrator is fully linear but has a rather large zero drift. Zero drift integrator fluctuations were adjusted carefully before each measurement. In order to increase measurement accuracy, a special technique was used [3]. Integrator zero drift values were measured before and after each measurement. The results were then interpolated to zero drift. The maximum available bench platform speed of $200 \mathrm{~mm} / \mathrm{sec}$ was used for coil measurements to decrease the influence of integrator zero drift.

A third order polynomial fit yields the results shown in Table 6 and Fig. 8 for the skew components of the integrated multipole moments (for an ID gap of $11.5 \mathrm{~mm}$ and for the symmetrical case).

Table 6. Integrated Multipole Moments

\begin{tabular}{|l|l|}
\hline Multipole n & Skew Component (an) \\
\hline 0 (dipole) & $-28 \mathrm{G}-\mathrm{cm} \pm 1.4$ \\
\hline 1 (quadrupole) & $24 \mathrm{G} \pm 5.1$ \\
\hline 2 (sextupole) & $8.6 \mathrm{G} / \mathrm{cm} \pm 5$. \\
\hline 3 (octupole) & $-17.2 \mathrm{G} / \mathrm{cm}^{2} \pm 12.6$ \\
\hline
\end{tabular}

The next set of measurements were performed to obtain skew " components of the integrated multipole moments for all range of gaps in the antisymmetric case. A typical horizontal field map measured with $B_{x}$-coil \#4 is shown in Fig. 9. A third order polynomial fit yields the results of skew component measurements, which are shown in Table 7. 
Table 7. Integrated Multipole Moments (Skew Components)

\begin{tabular}{|l|l|l|l|l|}
\hline gap (mm) & $\mathrm{a} 0(\mathrm{G}-\mathrm{cm})$ & $\mathrm{a} 1(\mathrm{G})$ & $\mathrm{a} 2(\mathrm{G} / \mathrm{cm})$ & $\mathrm{a} 3\left(\mathrm{G} / \mathrm{cm}^{2}\right)$ \\
\hline 11.5 & $-16 . \pm .76$ & $35 . \pm 4.2$ & $-3.2 \pm 6.2$ & $-56.4 \pm 23.6$ \\
\hline 14.5 & $-4.4 \pm .47$ & $6.3 \pm 2.6$ & $.05 \pm 3.8$ & $-13.5 \pm 14.4$ \\
\hline 20. & $-6.9 \pm .5$ & $-2.1 \pm 2.7$ & $-3.2 \pm 3.8$ & $-26.4 \pm 14.1$ \\
\hline 27. & $-9.5 \pm .76$ & $-26.7 \pm 4.1$ & $4.47 \pm 5.7$ & $22.3 . \pm 21.4$ \\
\hline 35. & $-10.4 \pm .7$ & $-32.6 \pm 3.7$ & $8.0 \pm 5.2$ & $-2.45 \pm 19.4$ \\
\hline 51. & $-7.1 \pm .5$ & $-32 . \pm 2.7$ & $-7.7 \pm 3.7$ & $4.9 \pm 13.9$ \\
\hline
\end{tabular}

These results fully satisfy the requirements of Table 1 for integrated skew components.

Typical RMS errors with $\mathrm{B}_{\mathrm{X}}$-coil \#4 for the first field integral measurements were 1-3 G-cm.

Coil measurement accuracy strongly depends on the correct choice of the integrator range and on accurate adjustment of the integrator zero drift.

\section{Conclusion}

Included here are some general remarks about the wedge-pole measurements taken. First, no adjustments were made to the end poles when the gap was changed. Also, the measurement results show that, in this case, we do not need end clamps. We shall not have any magnetic materials placed closer than $300 \mathrm{~mm}$ to the undulator, that is, in the region where the undulator might produce an influence on an external device (the fringing field at a distance of $100 \mathrm{~mm}$ from the end pole is about 2 Gauss). In our case the antisymmetric configuration allows us to satisfy the requirements for the both $J_{1}$ and $J_{2}$ simultaneously.

References

1. L. Burkel, R. Dejus, J. Maines, J. O'Brien, J. Pflüger, and I. Vasserman, ANL/APS/TB-12, March, 1993 
2. P. Zurmühl. "Praktische Mathematik" Springer Verlag, Heidelberg, 1965

3 J. Pflüger. Rev. Sci. 63(1), 1992, American Institute of Physics, p. 295 
Figure 1. Hall probe noise vs integration time

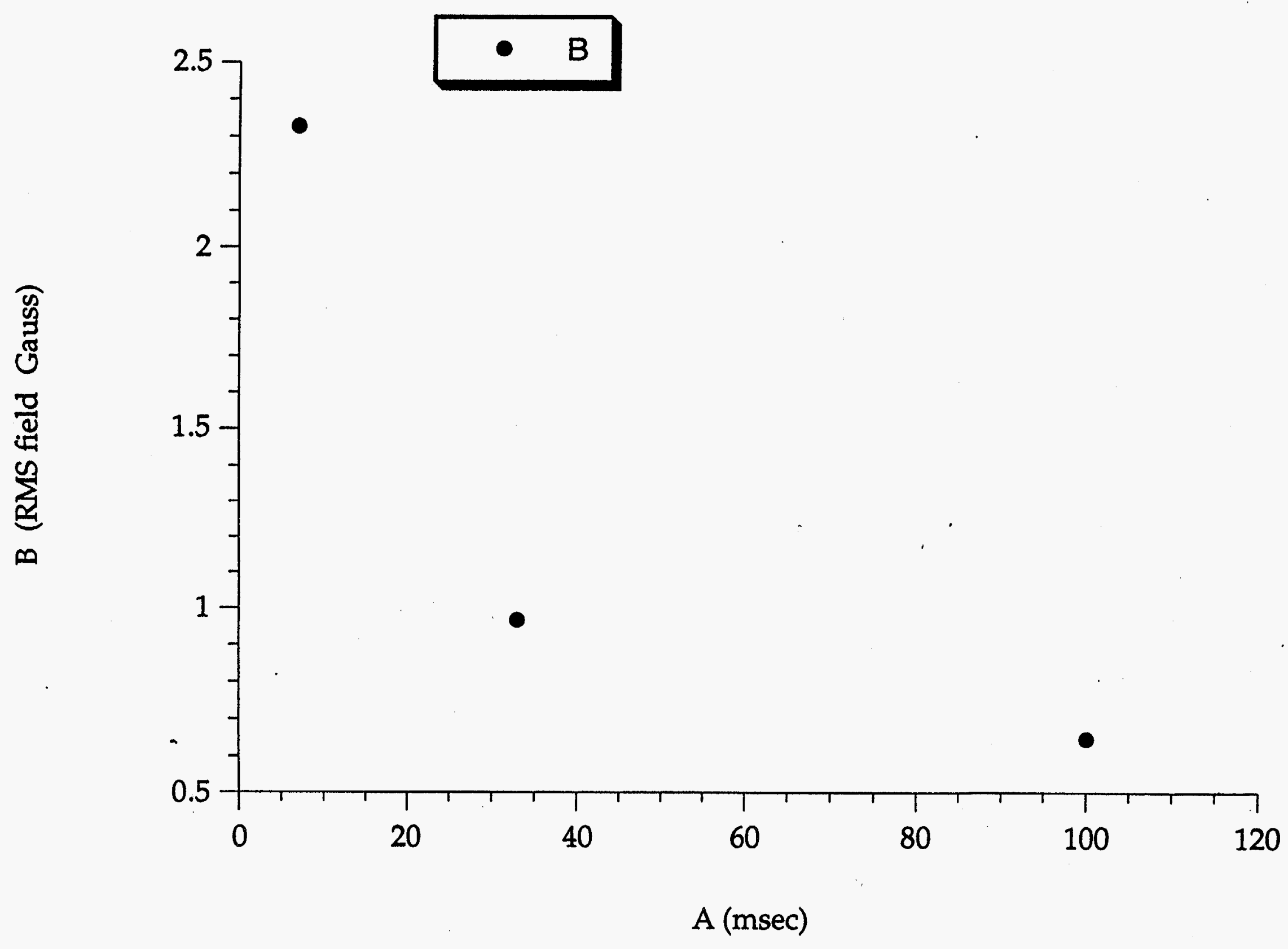


Figure 2. First Vertical Field Integral vs. $X$

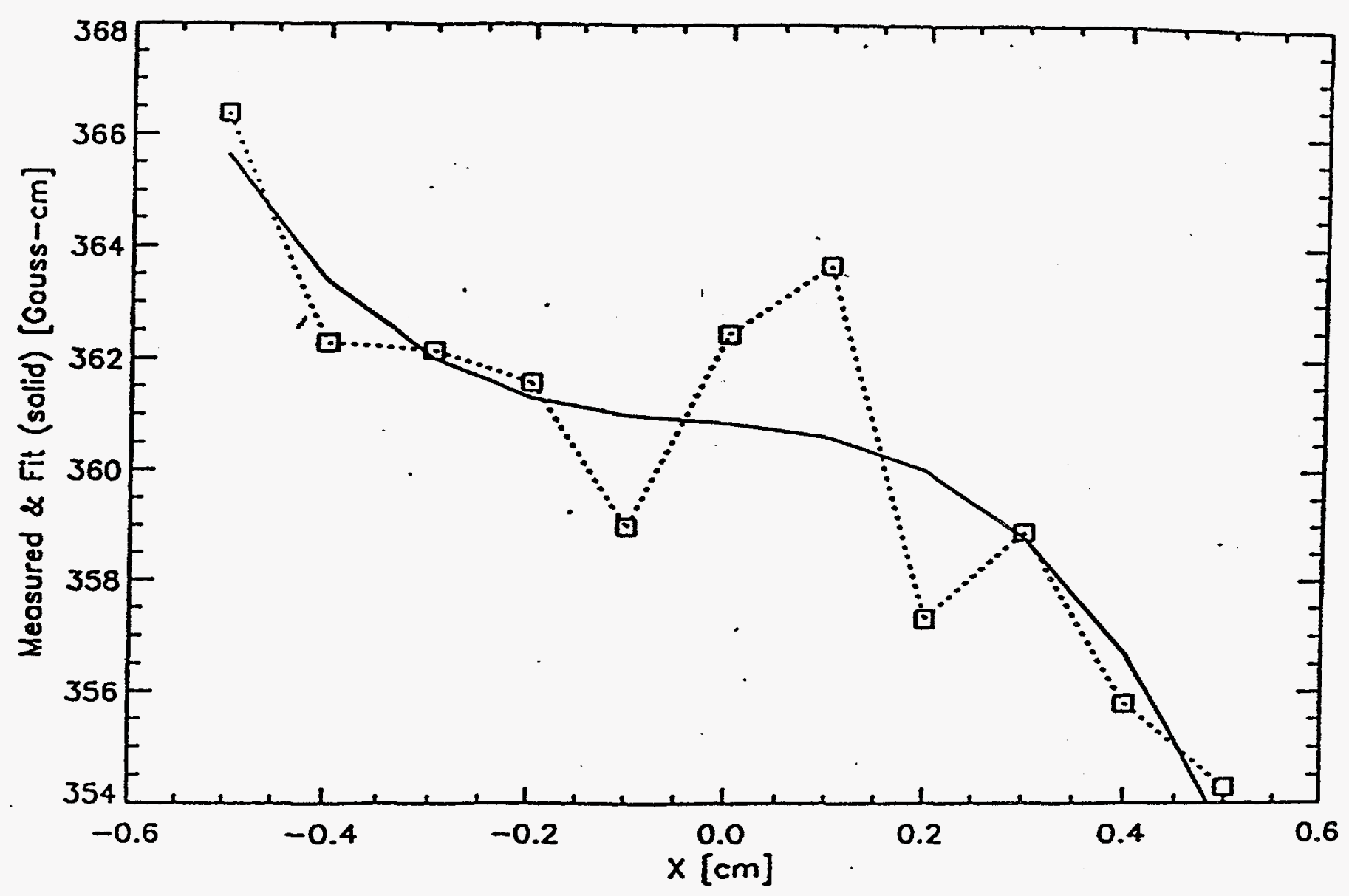


Figure J. First vercicial riela lintegral ( vis-cm)

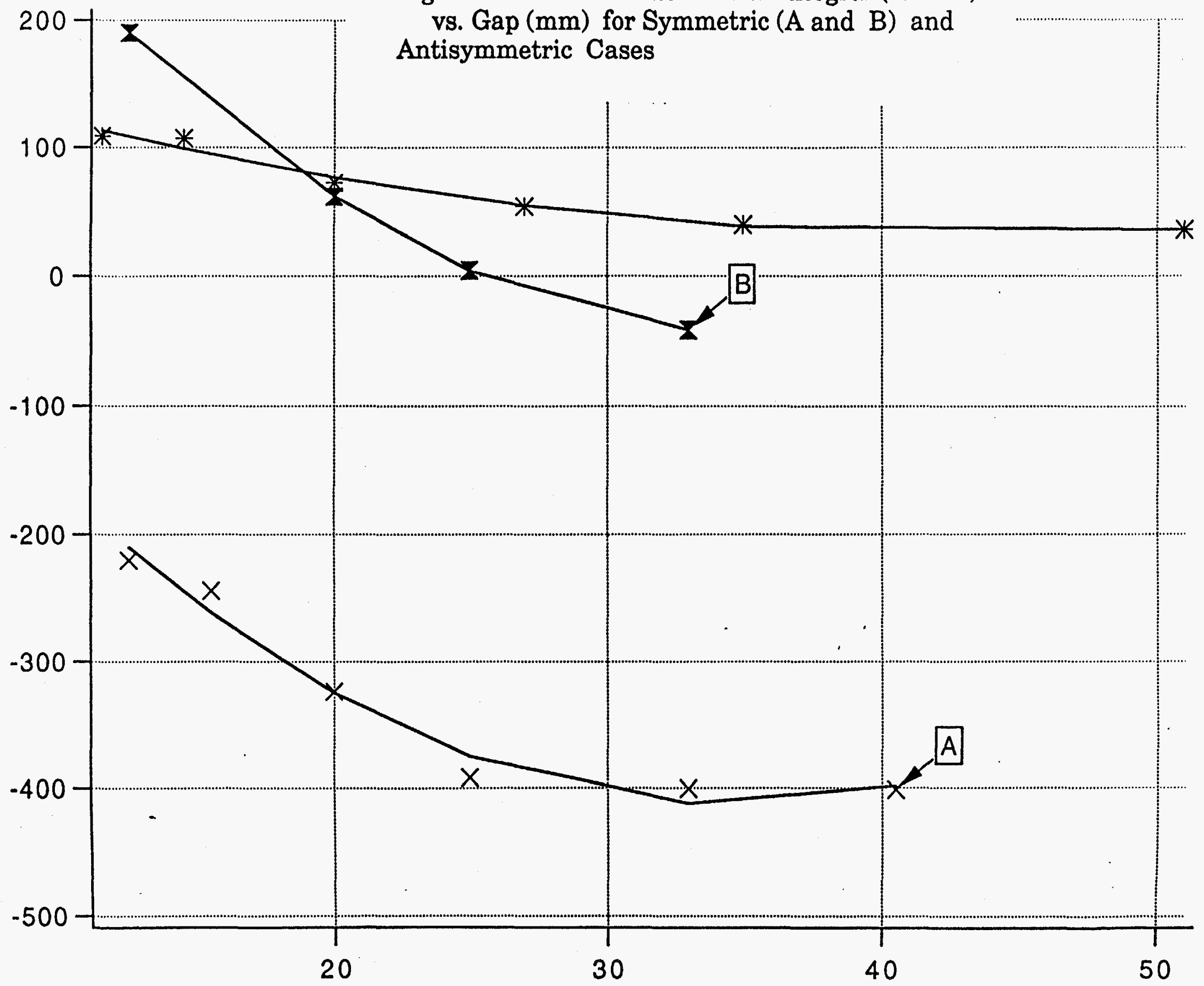




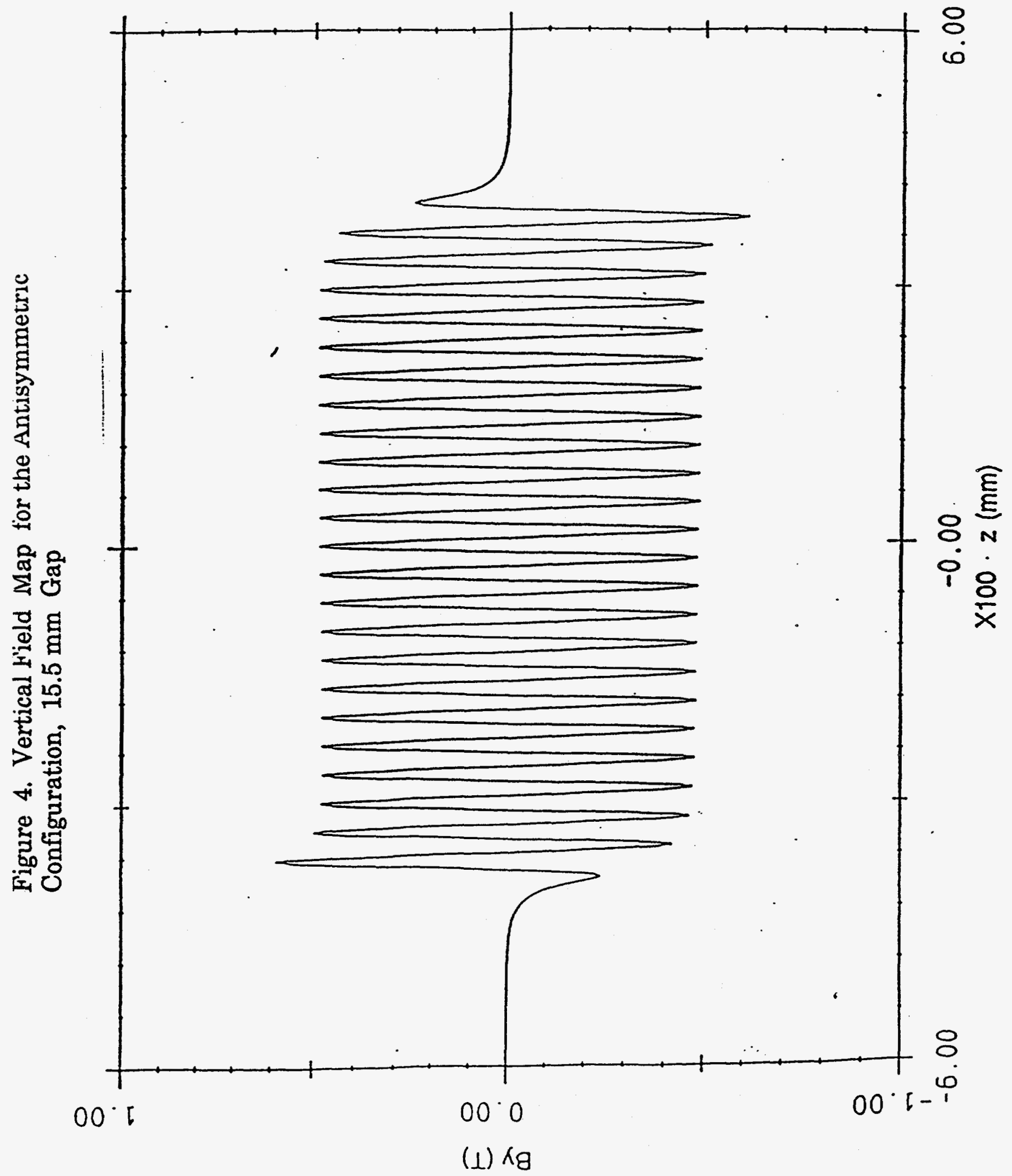




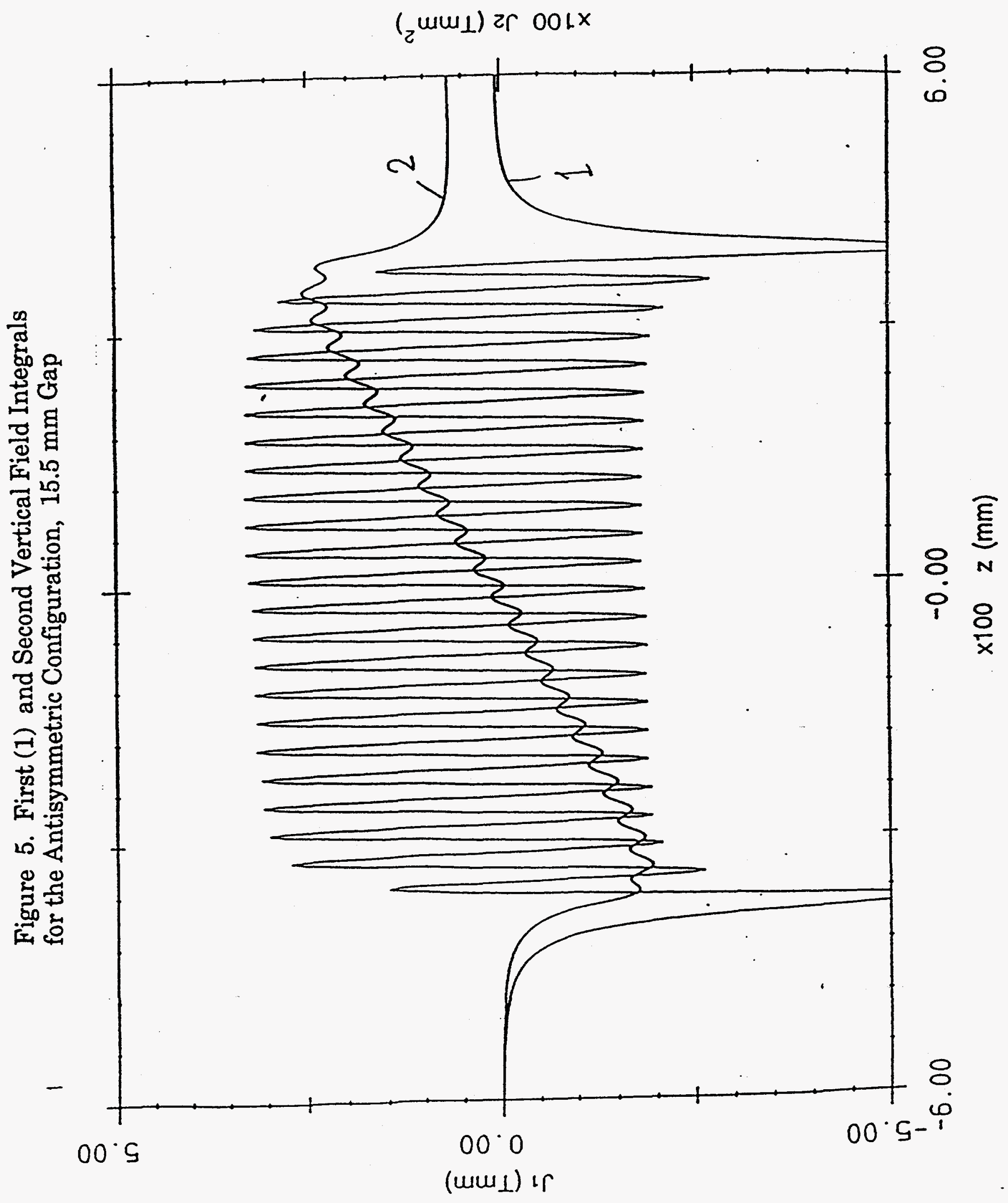



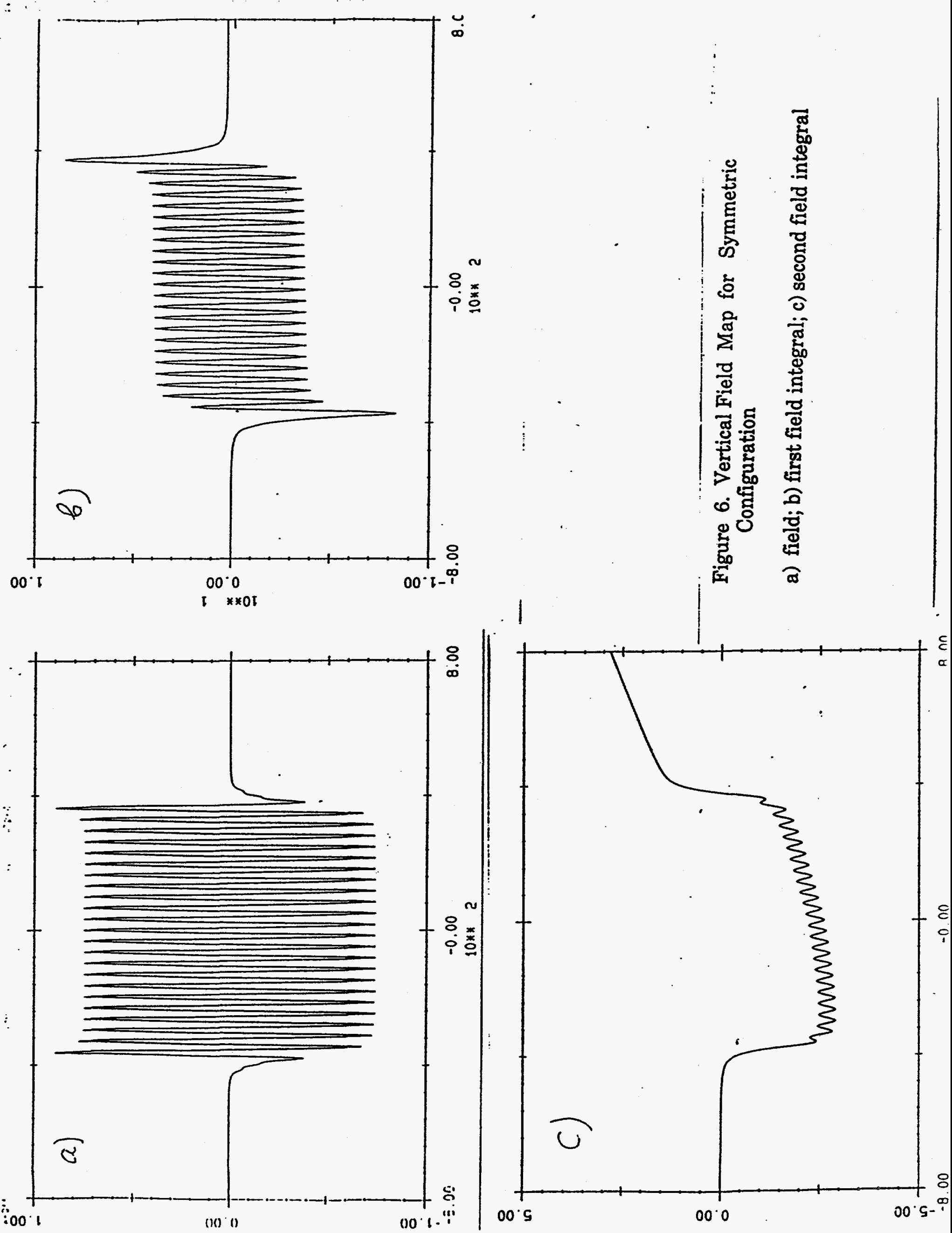


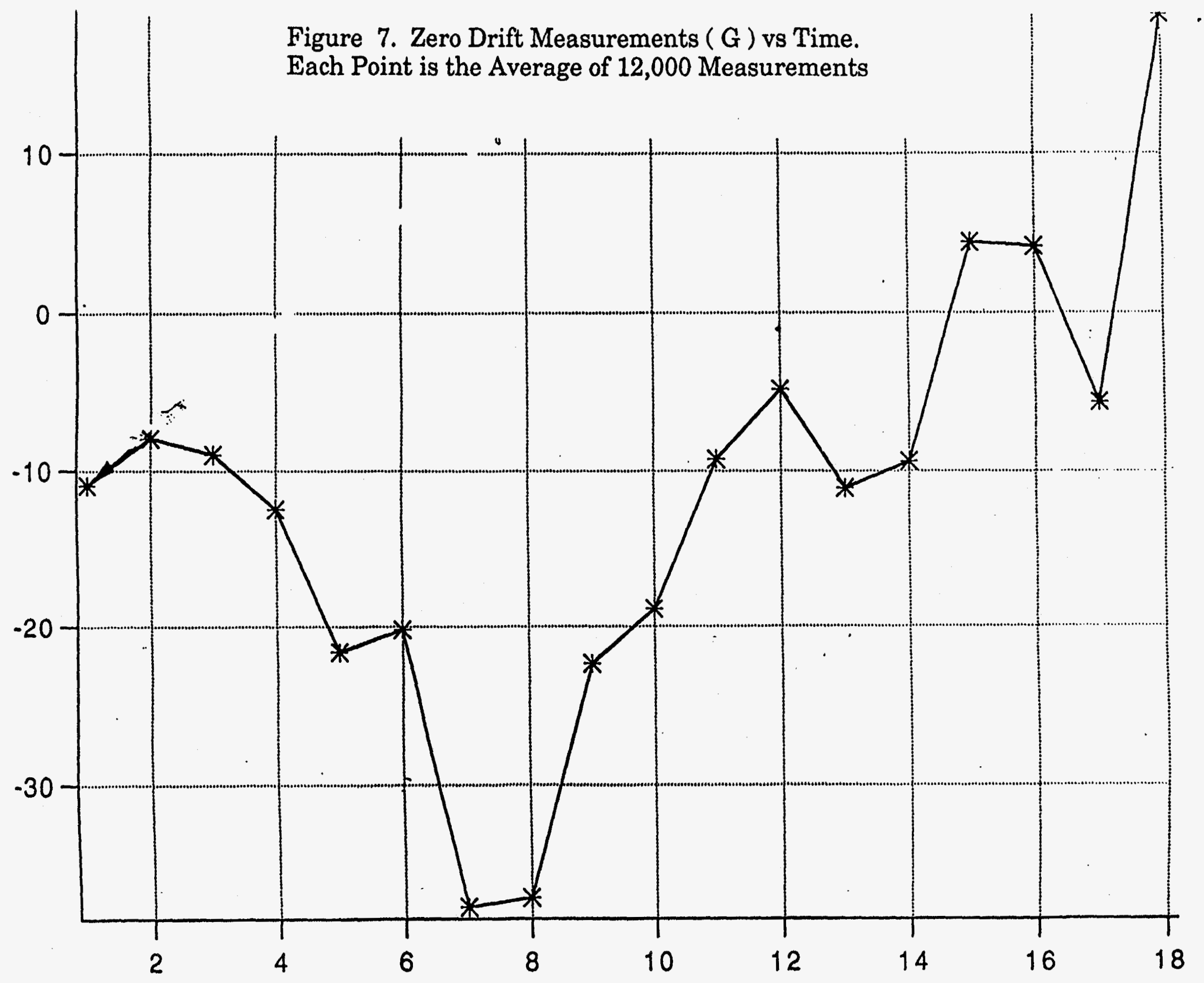


Figure 8. First Horizontal Field Integral vs $\mathrm{X}$

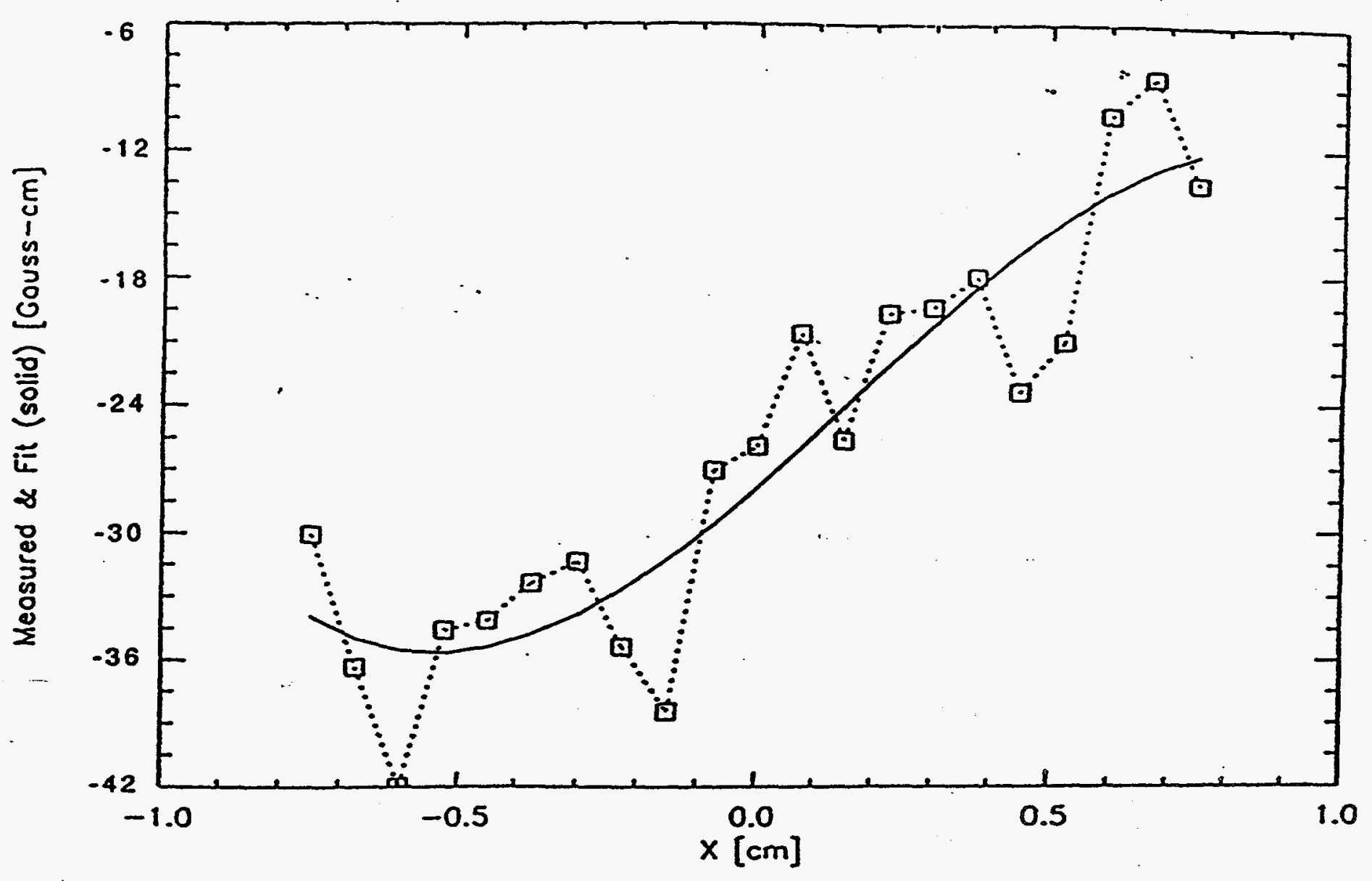




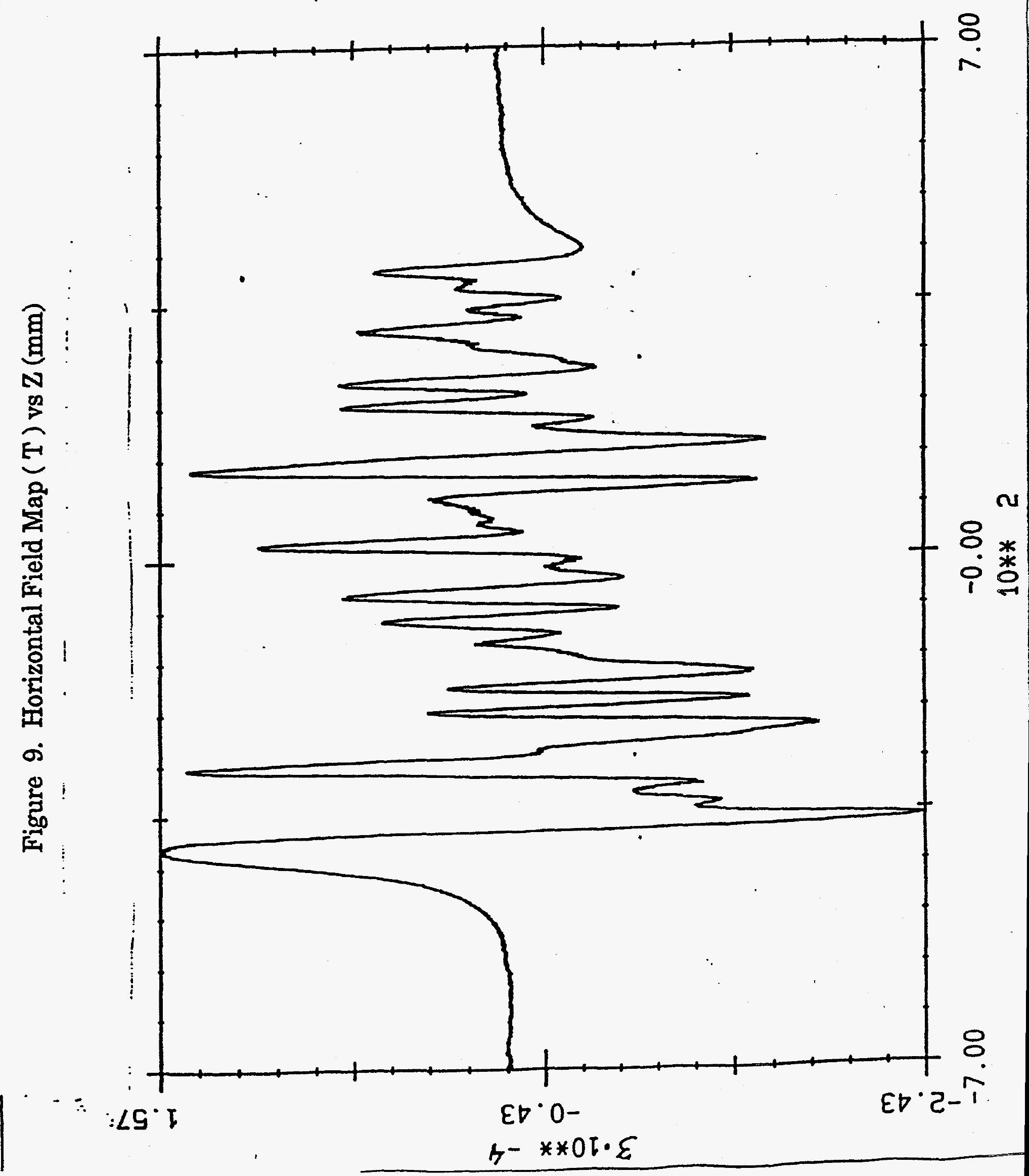

